\title{
A STUDY OF THE STRUCTURE OF WOOD DAMAGED BY ROT USING NUCLEAR MAGNETIC RESONANCE
}

\author{
Evgeny Onuchin* Andrey Medyakov Leonid Grunin Elena Sidorova \\ Volga State University of Technology, Yoshkar-Ola, Russia
}

Up-to-date production of fuel pellets has problems associated primarily with insufficient amount of raw material of high quality and its high cost. To increase the productivity of the pelletizing process, it is necessary to use defective trees including those affected by rot. In order to solve the problem of lack of strength of pellets, technical lignin is used as an additive. Various ways to determine the damage rate of wood by rot were investigated in order to know the amount of lignin needed to add to produce a product of high quality. The NMR relaxation method is characterized by simplicity of sample preparation, rapid acquisition of test results, accuracy, undemanding to personnel qualifications, relatively small equipment cost, and safety. In the course of the experiment, we obtained a curve of decrease of the free nuclear induction of the H-NMR sample containing a solid and a mobile phase. Graphs of the relative proportion of solid components and liquid phase shows the dependence of the content of cellulose and lignin in the sample and its moisture rate on the percentage of rotten wood.

Key words: Pellets, Rotten wood, Cellulose, Lignin, NMR relaxation

\section{INTRODUCTION}

At present, the potential reserves of fuel and energy resources in the Russian Federation are about 600 billion tons, but they are distributed unevenly across the territory. A significant part of them is located in the eastern regions, such as Siberia and the Far East. But Central, Southern and Western regions are characterized by a shortage of fuel and energy resources.

Besides, the country has the largest forest area, which is 774 million hectares or $45 \%$ of its territory. Fuel-and-energy-deficient regions also have large wood resources including those affected by rot, which are now not used at all. The reason why they are not used is the lack of technologies. Fortunately, there are some promising ways of using wood raw materials, such as production of pellets, which has a number of advantages in comparison with other types of fuel and energy resources:
1. Environmental safety;
2. High energy concentration;
3. Low amount of ash;
4. Relatively low cost;
5. Renewability (sustainability).

As a raw material for pellets, both industrial wood and wood waste are used: tree bark, sawdust, chips and other waste from the logging and wood processing industry. But as the volumes of harvesting are decreasing every year due to the reduction in stocks of industrial wood, the problem of making pellets from defective trees including rotted ones becomes topical.

The solution of this problem is possible when validating the technology for the production of pellets from wood with a high rate of damage by rot.
However, the problem of assessing the rate of damage of wood by rot takes place. The chemical composition of wood damaged by rot was studied by Falk [01], Komarov [02], Nikitin [03] and other researchers.

The analysis of the changes in the chemical composition of the wood that occur during rot given in the literature $[04,05,06]$ shows that in most cases the content of starch and pentosans in rotten wood reduces, and the content of substances soluble in water and benzene (extractives) increases. As for cellulose, lignin and other constituents of cell membranes, the amount of them depends on the type of fungus that causes rot.

However, lignin is a natural binder in pellets, and a reduction in its percentage in raw materials leads to a poor quality of final product. To eliminate this problem, it is proposed to additionally add lignin.

The content of rot depending on type of wood is different and, for its better use, it is important to know the percentage of rotten and sound wood.

Therefore, our task is to determine the relative proportions of moisture and solid components (cellulose, hemicelluloses, and lignin) in the tested wood samples in order to determine the rate of rot. The obtained data will allow us determining the amount of lignin, which should be added to wood raw material to obtain high-quality pellets.

There are several methods to measure the rate of damage to wood by rot, for example, chemical and ultrasonic methods.

Determination of the chemical composition of wood is associated with great difficulties due to the complex structure of the cell walls and the existence of strong bonds including chemical ones between the individual components. The main components of wood and other 
plant raw materials are high-molecular compounds (cellulose, lignin, hemicelluloses). Their separation can be achieved only after certain chemical reactions that can lead to significant change of the identified components and to their partial loss. In other words, the chemical analysis of wood is to some extent conditional.

The ultrasonic method refers to non-destructive methods of control. The main advantage of this method is the ability to evaluate the parameters and working properties of the object using control methods that do not involve cutting out samples. The study is carried out in operating conditions. This makes it possible to partially avoid material and time costs. The disadvantage is the high cost of equipment.

At the same time, in many areas of on-line and at-line control processes, the method of nuclear magnetic relaxation (NMR relaxation) is used.

This method has been successfully applied in food $[07,08]$, polymeric $[09,10,11,12]$, textile [13], pulp and paper $[14,15]$ industries, in studying the aging processes of materials [16], in medical and ecological researches $[17,18]$.

The advantages of the NMR relaxation method include simplicity of sample preparation, rapid analysis results (several minutes), accuracy, low requirements for per- sonnel qualifications, relatively small equipment cost, simplicity of operation, and safety.

In this paper we proposed a method for the experimental determination of the rate of wood damage by rot using the NMR relaxation method.

\section{MATERIALS AND METHODS}

In order to simulate the degree of damage to wood at various stages of rot development, samples of sawdust of spruce, pine and birch (5 samples each) with a different percentage of sound and rotten wood were prepared (Table 1). For this purpose, we took round tree cross-section disks of air-dry sound wood (with moisture content of $20-30 \%$ ) and ones damaged by rot (Figure 1). Rot in the wood was determined visually. Then the cross-section disks were crushed to particles not larger than $2 \mathrm{~mm}$. With the help of analytical scales with a measurement resolution of not more than $0.1 \mathrm{mg}$, the samples were weighed and after that placed in identical test tubes with a diameter of $10 \mathrm{~mm}$ (Figure 2). The tubes were tightly closed and mounted in the sensor of the NMR analyzer. The sample preparation procedure was of low labor input and was reduced to placing the analyzed wood in a test tube and installing the latter into the sensor of the device.

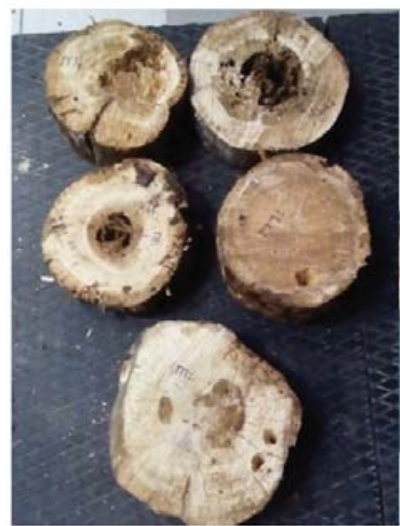

A) spruce

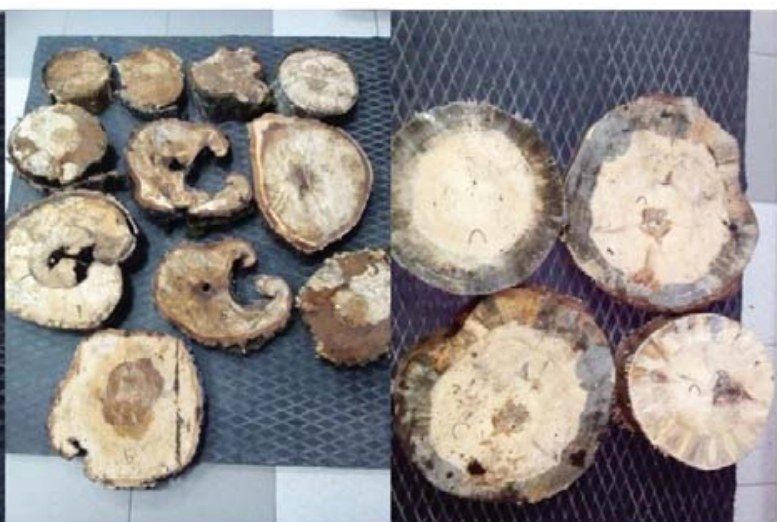

B) birch
C) pine

Figure 1: Samples of wood for research

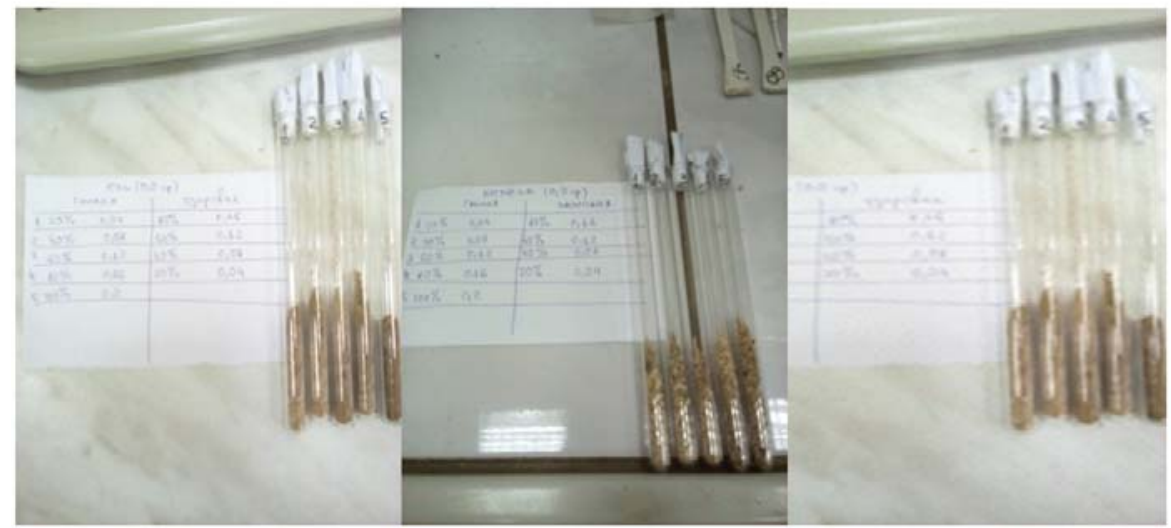

A) spruce

B) birch

C) pine

Figure 2: Sawdust samples with different percentages of rotten wood 
The measurements were carried out at an ambient air temperature of $20-25^{\circ} \mathrm{C}$ and atmospheric pressure.

Parameter measurements were performed using NMR analyzer «Spin Track» manufactured by Resonance Systems Ltd. located in Yoshkar-Ola, Russian Federation (Figure 3).

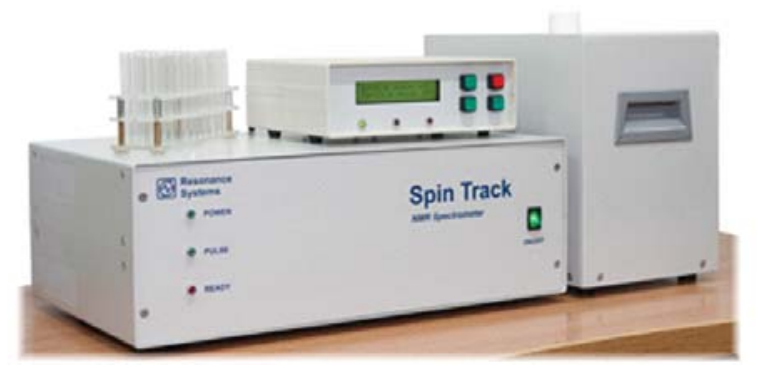

Figure 3: NMR analyzer

Table 1: Percentage of rotten and sound wood in samples

\begin{tabular}{|c|c|c|}
\hline Sample No & Rotten wood, $\%$ & Sound wood, \% \\
\hline 1 & 20 & 80 \\
\hline 2 & 40 & 60 \\
\hline 3 & 60 & 40 \\
\hline 4 & 80 & 20 \\
\hline 5 & 100 & 0 \\
\hline
\end{tabular}

\section{RESULTS}

The following curve was obtained in the course of the experiment when measuring the signal of free nuclear induction decrease (Figure 2), which can be approximated by the sum of two functions:

$$
a(t)=a_{s} \exp \left(-\left(t / T_{2 s}\right)^{2}\right)+a_{l} \exp \left(-t / T_{2 l}\right)
$$

where $t$ - time, us; $T$ - amplitude,a.u.

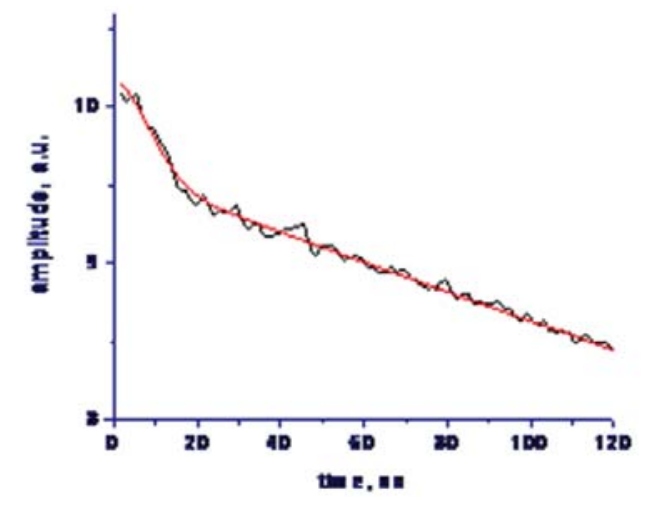

Figure 4: Decrease of free induction of H-NMR-sample containing a solid and a mobile phase
Components with index s correspond to the solid phase of the sample (cellulose, hemicelluloses, and lignin)

Components with index I correspond to the mobile (liquid) phase of the sample, which is water in macropores. Amplitudes reflect the number of components.

With an increase in the percentage of rotten wood in the sample, the content of cellulose and lignin decreases (Figure 5) and the humidity increases (Figure 6). In samples with a rotten wood content of 20 to $60 \%$, cellulose and lignin decompose slowly, and humidity increases slightly. A jump is observed with an increase in the proportion of rotten wood from 60 to $100 \%$.

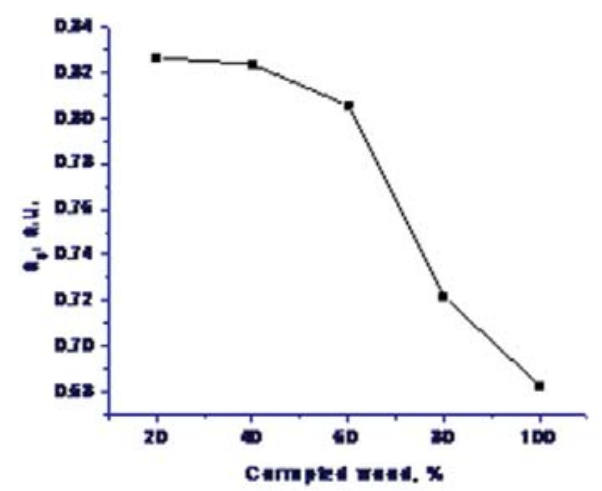

Figure 5: Relative proportion of solid components (cellulose, hemicelluloses, and lignin)

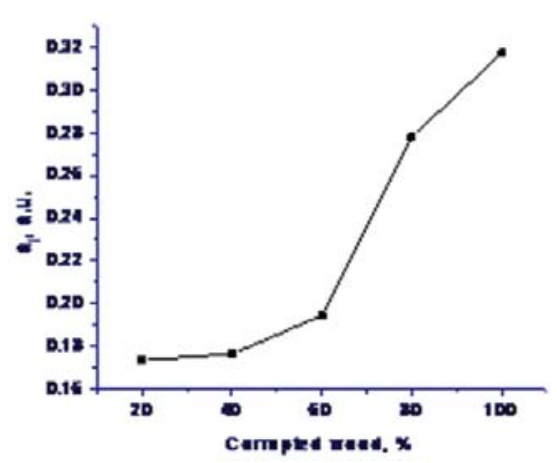

Figure 6: Relative proportion of mobile (liquid) phase (water in macropores)

The results presented were obtained for spruce samples, but similar characteristics were also found for birch ones. The simulation veracity is confirmed by studies presented in [19] and [20]. In accordance to them, the physical properties of wood (density and humidity) were investigated without taking into account the species of rotting fungus. At the same time, the density was determined by the method presented in the State Standard 1148665 (GOST 11486-65), and the humidity was determined in accordance with the State Standard 11491-65 (GOST 11491-65). 


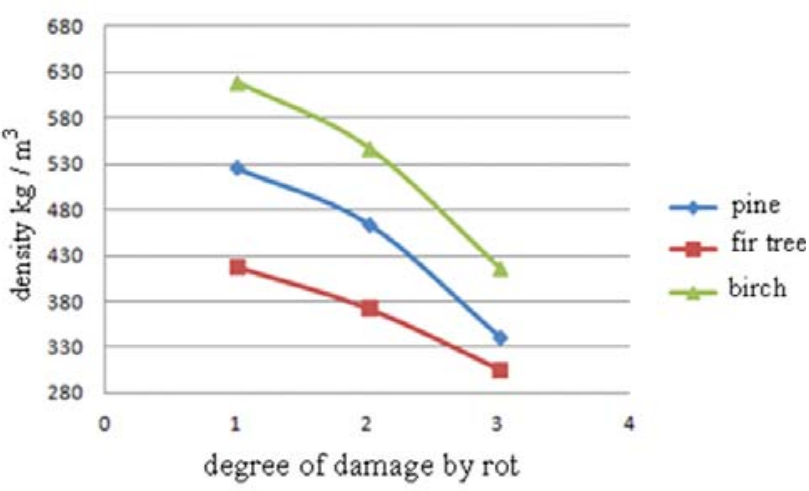

Figure 7: Density of wood, depending on the degree of damage to its rot [21]

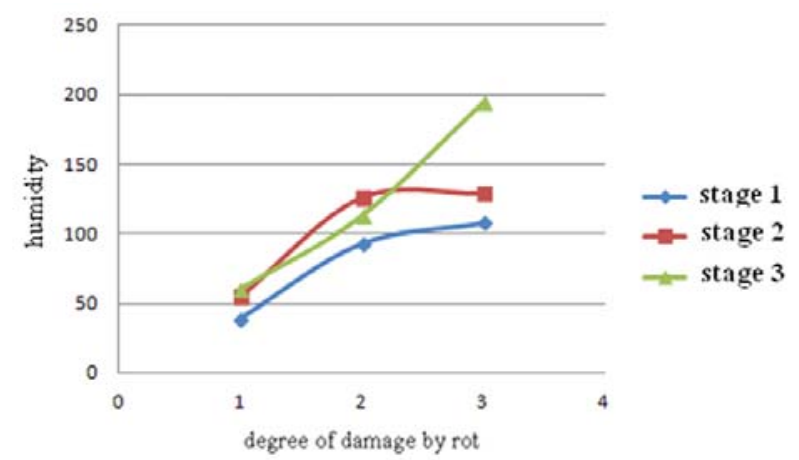

Figure 8: Humidity of wood, depending on the degree of damage to its rot [21]

\section{DISCUSSION}

It is known that the process of rot and destruction of wood by fungi characterized by unique set of enzymes, is accompanied by the loss of biological properties and technical qualities, for example, by changing the humidity.

The maximum level of humidity of the stem wood is limited by the total volume of the cell cavities and intercellular spaces. When rotting wood, its cells are destroyed forming additional internal cavities; the structure of rotten wood becomes friable and porous as the rot process develops; at the same time the strength of the wood is sharply reduced.

For these reasons, the moisture content of wood rot can reach very high values, at which its burning becomes ineffective. Increased porosity of rotten wood makes it very hygroscopic being outdoors, and it is quickly moistened.

Based on the results of the experiment, it is possible to validate the studies [04, 05, and 06] performed to determine the chemical composition of the wood for different stages of rotting damage (Figure 5). The more percentage of rotten wood is in samples, the less the proportion of solid components (cellulose, lignin, and hemicelluloses) they have.

Most likely, this is due to the destruction of the medullary rays and tori in the trees by hyphae of fungi and the ap- pearance of perforations in the walls of cells, as well as the reduction in their thickness. As a result, the amount of wood substance per tree volume unit is reduced and the volume of voids increases. In addition, during the process of rotting the components of the cell walls are destroyed. All this leads to an increase in the porosity of wood and a decrease in its density, as shown in Figure 7.

An increase in the moisture content of samples with an increase in the percentage of rotten wood in them (Figure 6) is associated with a change in the structure of wood due to the destruction of its walls by hyphae of fungi. They create additional paths for the passage of liquids, which leads to greater absorption of water by rotten wood. This is confirmed by studies given in [19], the results of which are shown in Figure 8.

Thus, we can conclude that the NMR relaxation method can be uniquely used to quickly assess the rate of damage of wood by rot.

\section{CONCLUSIONS}

Experiments performed using simulated samples showed that with increasing percentage of rotten wood, the content of cellulose and lignin decreases and humidity increases.

On the basis of the study carried out, it can be concluded that the NMR relaxation method allows a rapid and qualitative assessment of the relative proportions of solid and mobile components in wood raw materials.

The obtained data will allow determining the amount of lignin, which can be added to wood raw materials for obtaining high-quality fuel pellets.

\section{REFERENCES}

1. Falk R. Die Lenzites-faule des Coniferholzes. Molier's Hausschwamm-forschungen. Heft 3, 1909.

2. Nikitin N.I., Solechnik N.Ya., Komarov F.P. (1931) Chemical technology of wood. N.I. Nikitina (Eds.), State scientific technical publishing house, Leningrad,

3. Nikitin, V.M. (1978) Chemistry of wood and cellulose. V.M. Nikitin, A.V. Obolenskaya, V.P. Scheglov (Eds.), Forest industry, Moscow.

4. Nikitin N.I. (1962) Chemistry of wood and cellulose. Publishing house of Academy of Sciences SSSR. Moscow-Leningrad.

5. Vanin S.I. (1931) The rot of the tree, its causes and measures of struggle. Moscow-Leningrad.

6. Ripachek, V. (1967) Biology of wood-destroying fungi. Publishing house "Forest industry". Moscow.

7. GOST 53158-2008 (ISO 8292:2008). (2009) Vegetable oils, animal fats and processed products. Determination of solid fat content by pulsed nuclear magnetic resonance. Standartinform, Moscow. 
8. http://www.bruker.com/products/mr/td-nmr/minispec-mq-polymer-research-analyzer /learn-more. html, accessed on 2017-12-25.

9. https://www.bruker.com/fileadmin/user upload/8PDF-Docs/MagneticResonance/NMR/ brochures/ Crystallinity_app_note_T149125.pdf, accessed on 2017-12-25.

10. Blyumih, B. (2011) Fundamentals of NMR for scientists and engineers. B. Blyumih. (Eds.), Tehnosfera, Moscow.

11. Hertlein, C., Strobl G., Saalwachter K. (2006) Lowfield NMR studies of polymer crystallization kinetics: Changes in the melt dynamics. Polymer. no. 47 (20), 7216-7221.

12. M. Mauri, Y. Thomann, H. Schneider, K. Saalwächter (2008) Spin Diffusion NMR at Low Field for the Study of Multiphase Solids. Solid State Nuclear Magnetic Resonance. no 34, 125-141 DOI: ...10.1016 / j.ssnmr.2008.07.001.

13. https://www.oxford-instruments.com/industries-and-applications/textiles/spin-finish-on-artificial-fibres, accessed on 2017-12-25.

14. Grunin Y.B., Grunin L.Y., Nikol'Skaya E.A., Talancev V.I. (2012) Microstructure of cellulose: NMR relaxation study. Polymer Science. Series A., vol 54, no 3, 201-208.

15. Grunin Y.B., Grunin L.Y., Nikol'Skaya E.A., Talancev V.I., Gogelashvili G.Sh. (2015) Features of the structural organization and sorption properties of cellulose. Polymer Science. Series A, vol 57, Iss. 1, 43-51.
16. Pfleiderer B, Xu P, Ackerman JL, Garrido L. (1995) Study of aging of silicone rubber biomaterials with NMR. Journal of Biomedical Materials Research, no. 29, 1129-1140.

17. Persson B.R., Malmgren L., Salford L. (2012) Studies of H-NMR Relaxation Dispersion in Human Brain-tissue Samples: Implications for Magnetic Resonance Relaxation Dispersion Imaging (MARDI). Acta Scientiarum Lundensia, no 1, 1-22.

18. Vanhamme L., Sundin T., Van Huffel S., Van Hecke P. (2001) MR spectroscopy quantitation: a review of time-domain methods. NMR in Biomedicine, no 14, 233-246.

19. Vanin S. I.,. Bazhenov V. A, Vikhrova E. V. I On the moisture content of healthy and mushroomed wood in the trunks of growing trees / S. I. Vanin, V. A. Bazhenov, E. V. Vikhrova / / Tr. Institute of Forest of the Academy of Sciences of the USSR, 1953, volume 4.

20. Perelygina L. M., Pevtsov, A. H. About influence of the initial stage of rotting on physical and mechanical properties of wood of pine, birch, persimmon. / L. M. Perelygina, A. H. Pevtsov, // Proceeding «To the question of replacing scarce wood species», 1933. The publication CNIIMOD.

21. Wood and wood materials. Krasnoyarsk, 1974 